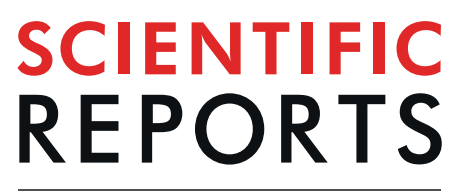

\title{
Changes in metabolic syndrome and its components and the risk of type 2 diabetes: a nationwide cohort study
}

\author{
Min-Kyung Lee ${ }^{1}$, Kyungdo Han², Mee Kyoung Kim³ ${ }^{3}$ Eun Sil Koh ${ }^{4}$, Eun Sook Kim ${ }^{5}$, \\ Ga Eun Nam ${ }^{6} \&$ Hyuk-Sang Kwon ${ }^{3 *}$
}

We investigated the relationship of changes in Metabolic syndrome (MetS) and its components with the risk of type 2 diabetes (T2D) in South Korea. Records of 10,806,716 adults aged $\geq 20$ years without a history of T2D between 2009 and 2015 were retrieved from database of the South Korean National Health Insurance Service and analyzed. Changes in metabolic components were monitored over a twoyear period with follow-up occurring at an average of 4.087 years. During the follow-up period, 848,859 individuals were diagnosed with T2D. The risk of diabetes was lowered with a decrease in the number of MetS components at baseline and the second visit ( $p$ for trend $<0.0001$ ). Multivariable-adjusted HRs for incident diabetes were 0.645 among individuals with reduced number of MetS components, 0.54 for those with improvement in elevated fasting glucose, 0.735 for those with improvement in elevated triglycerides, 0.746 for those with improvement in elevated blood pressure, 0.763 for those with improvement in reduced HDL-cholesterol, and 0.92 for those with improvement in abdominal obesity compared with those manifesting them at both time points. In conclusion, changes in metabolic syndrome and its components were significantly associated with the development of T2D. Improvement in MetS and its components attenuated the risk of diabetes.

Type 2 diabetes (T2D) is a chronic disease resulting from a complex interaction between heredity and environment, along with other risk factors ${ }^{1}$. Insulin resistance, obesity, and behavioral factors such as physical activity, diet, smoking, alcohol consumption, and body weight are important risk factors for $\mathrm{T}_{2} \mathrm{D}^{2}$. Increasing incidence of diabetes and related complications imposes a heavy health burden ${ }^{3}$. Thus, investigations should focus on effective interventions and preventive measures for the disease.

Metabolic syndrome (MetS) was conceptualized based on a constellation of risk factors, such as elevated fasting plasma glucose (FPG), atherogenic dyslipidemia, elevated blood pressure, and abdominal obesity, in individuals susceptible to cardiovascular disease (CVD) and $\mathrm{T}^{2} \mathrm{D}^{4}$. Guidelines for clinical and epidemiological management of MetS have been developed using readily available clinical variables ${ }^{5}$. A number of studies have demonstrated a strong association of MetS and its components with the risk of incident T2 $\mathrm{D}^{6-8}$. Thus, clinical implications of MetS should be focused on multifactorial interventions to reduce the risk of T2 ${ }^{9}$. All individuals deserve long-term management of MetS and follow-up in the clinical setting ${ }^{10}$. However, cohort studies that correlate the development of T2D with changes in MetS and its components are lacking. Thus, the objective of this nationwide population-based cohort study was to investigate association of changes in MetS and its components with risk of T2D in South Korea.

\footnotetext{
${ }^{1}$ Division of Endocrinology and Metabolism, Department of Internal Medicine, Myongji Hospital, Hanyang University College of Medicine, Gyeonggi-do, Republic of Korea. ${ }^{2}$ Department of Medical Statistics, College of Medicine, The Catholic University of Korea, Seoul, Republic of Korea. ${ }^{3}$ Division of Endocrinology and Metabolism, Department of Internal Medicine, Yeouido St. Mary's Hospital, College of Medicine, The Catholic University of Korea, Seoul, Republic of Korea. ${ }^{4}$ Division of Nephrology, Department of Internal Medicine, Yeouido St. Mary's Hospital, College of Medicine, The Catholic University of Korea, Seoul, Republic of Korea. ${ }^{5}$ Division of Endocrinology and Metabolism, Department of Internal Medicine, Incheon St. Mary's Hospital, College of Medicine, The Catholic University of Korea, Seoul, Republic of Korea. ${ }^{6}$ Department of Family Medicine, Korea University Anam Hospital, Korea University College of Medicine, Seoul, Republic of Korea. *email: drkwon@catholic.ac.kr
} 
Participants aged $\geq 20$ years

$\mathrm{N}=17,539,992$ between 2009 and 2010 (baseline examination) $\mathrm{N}=19,393,445$ between 2011 ant 2012 (2 ${ }^{\text {nd }}$ visit)

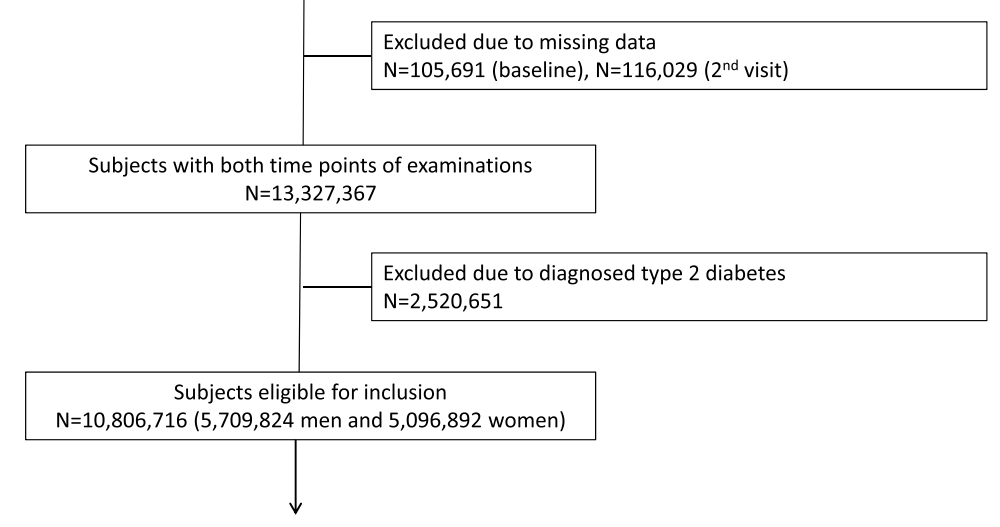

Followed to the date of developed type 2 diabetes or until the end of 2015

Figure 1. Flow chart of patient enrollment. Patients were followed by the development of type 2 diabetes or until the end of 2015.

\section{Methods}

Study subjects. We used the database provided by the National Health Insurance Service (NHIS), the single insurer managed by the South Korean government ${ }^{11}$. The NHIS public database contains data pertaining to health care utilization, health screening, socio-demographic variables, and mortality of the whole population (more than 51 million) of South Korea including information obtained from medical treatment and health screening records. Individuals enrolled in the NHIS are recommended to undergo standardized health examinations every two years. Disease diagnoses in the database are classified according to the International Classification of DiseaseTenth Revision-Clinical Modification (ICD-10-CM) codes. This study was approved by the NHIS inquiry commission, and the Institutional Review Board of The Catholic University of Korea (No. SC18ZESIO047).

From this cohort, the subjects aged $\geq 20$ years who had participated in health examinations between 2009 and 2010 and were reexamined in 2011 and 2012 were selected. 17,539,992 individuals received health examinations at baseline and 19,393,445 individuals were examined two years later. We then excluded 105,691 individuals at baseline examination and an additional 116,029 at the second visit who had missing data involving at least one variable. Among 13,327,367 individuals with both time points of health examinations, we excluded 2,520,651 who had been diagnosed with T2D. Finally, a total of 10,806,716 individuals (5,709,824 men and 5,096,892 women) were included in this study. They were followed up to monitor the development of T2D or until the end of 2015 with a median follow-up duration of 4.087 years (Fig. 1).

Diagnosis of type 2 diabetes. The NHIS database (DB) represents the entire Korean population ${ }^{12}$. Among the total datasets of the NHIS DB, we used qualifications, claims, the health check-up DB, and death information. Due to the inevitable limitations of the claim DB, T2D was defined operationally ${ }^{13}$. T2D from the claim DB was categorized based on the ICD-10 code of T2D (E11-E14) as principal diagnosis or up to a fourth additional diagnosis and at least one antidiabetic drug prescribed in a given year. T2D from health check-up DB was defined based on FBG (undiagnosed diabetes) or the ICD-10 code of T2D with a claim for antidiabetic medication. To validate the information accuracy, an expert committee from the Korean Diabetes Association reviewed the dataset regularly. The committee decided on the suitability of the dataset and reviewed the results of the analysis.

Definition of metabolic syndrome. MetS was defined by a combination of abdominal obesity, impaired fasting glucose, atherogenic dyslipidemia, and elevated blood pressure. Revised NCEP ATP III criteria ${ }^{10,14}$ require at least three of the following components: (1) abdominal obesity (waist circumference [WC] $\geq 90 \mathrm{~cm}$ for men, or $\geq 85 \mathrm{~cm}$ for women $)^{15}$; (2) triglycerides $\geq 150 \mathrm{mg} / \mathrm{dL}$, and/or drug treatment for elevated triglycerides; (3) high-density lipoprotein (HDL)-cholesterol $<40 \mathrm{mg} / \mathrm{dL}$ for men, or $<50 \mathrm{mg} / \mathrm{dL}$ for women; (4) systolic blood pressure (BP) $\geq 130 / 85 \mathrm{mmHg}$ or antihypertensive medication treatment, and/or a history of hypertension; and (5) $\mathrm{FPG} \geq 100 \mathrm{mg} / \mathrm{dL}$, and/or treatment with medications for T2D.

Measurement of covariates. Height, weight, WC, and BP was measured during regular medical check-ups in accordance of the heath checkups implementation standard ${ }^{16}$. Brachial BP was measured after 5 minutes of rest in a sitting position. The BP measurement was repeated if the first measurement exceeded $120 / 80 \mathrm{~mm} \mathrm{Hg}$. Body mass index (BMI) was calculated as weight in kilograms divided by height in meters squared $\left(\mathrm{kg} / \mathrm{m}^{2}\right)$. Serum samples for measuring FPG, total cholesterol (TC), HDL-cholesterol, and triglyceride levels were obtained after overnight fasting before each examination. Detailed histories of smoking status, alcohol consumption, physical activity, and past medical history were obtained using a self-administered questionnaire. Based on smoking status, subjects were classified into non-smokers, ex-smokers, or current smokers. Individuals who consumed $\geq 30 \mathrm{~g}$ of alcohol per day were defined as heavy alcohol drinkers. Physical activity was categorized by the frequency of 


\begin{tabular}{|c|c|c|c|}
\hline & \multicolumn{2}{|c|}{ New-onset type 2 diabetes } & \multirow[b]{2}{*}{$p$-value } \\
\hline & No & Yes & \\
\hline Participants, $n$ & 9888223 & 848859 & \\
\hline Age (years) & $47.19 \pm 13.06$ & $57.6 \pm 12.3$ & $<0.0001$ \\
\hline Male (\%) & $423272(49.86)$ & $5286552(53.09)$ & $<0.0001$ \\
\hline Body mass index $\left(\mathrm{kg} / \mathrm{m}^{2}\right)$ & $23.54 \pm 3.1$ & $24.76 \pm 3.26$ & $<0.0001$ \\
\hline Waist circumference $(\mathrm{cm})$ & $79.47 \pm 8.89$ & $83.82 \pm 8.73$ & $<0.0001$ \\
\hline Total cholesterol (mg/dL) & $195.44 \pm 35.22$ & $194.33 \pm 39.71$ & $<0.0001$ \\
\hline Triglycerides (mg/dL)*, & $103(72-154)$ & $122(85-179)$ & $<0.0001$ \\
\hline HDL cholesterol (mg/dL) & $55.81 \pm 14.56$ & $53.64 \pm 14.41$ & $<0.0001$ \\
\hline Systolic blood pressure (mmHg) & $120.91 \pm 14.28$ & $126.82 \pm 15.2$ & $<0.0001$ \\
\hline Diastolic blood pressure $(\mathrm{mmHg})$ & $75.6 \pm 9.76$ & $77.77 \pm 9.93$ & $<0.0001$ \\
\hline Fasting plasma glucose $(\mathrm{mmol} / \mathrm{l})$ & $92.31 \pm 10.61$ & $100.9 \pm 13.42$ & $<0.0001$ \\
\hline Metabolic syndrome (\%) & $1857347(18.65)$ & $349302(41.15)$ & $<0.0001$ \\
\hline Elevated fasting glucose (\%) & $2218827(22.28)$ & $322049(37.94)$ & $<0.0001$ \\
\hline Elevated triglycerides (\%) & $2935369(29.48)$ & $386278(45.51)$ & $<0.0001$ \\
\hline Reduced HDL-cholesterol (\%) & $2305559(23.15)$ & $317150(37.36)$ & $<0.0001$ \\
\hline Elevated blood pressure (\%) & $3692653(37.08)$ & $492302(58)$ & $<0.0001$ \\
\hline Abdominal obesity (\%) & $1556385(15.63)$ & $240280(28.31)$ & $<0.0001$ \\
\hline Current smoker (\%) & $269527(19.55)$ & $2308698(23.4)$ & $<0.0001$ \\
\hline Alcohol drinker (\%) & $582198(5.86)$ & $51101(6.04)$ & $<0.0001$ \\
\hline Regular physical activity (\%) & $5513578(55.8)$ & $702578(48.85)$ & $<0.0001$ \\
\hline
\end{tabular}

Table 1. Baseline Clinical Demographics of Participants Diagnosed with Type 2 Diabetes During Follow-up. Data are presented as mean \pm standard deviation (SD) or proportions (\%). *Geometric mean.

$\geq 20$ minutes of strenuous exercise. Hospitals conducting health examinations were certified by the NHIS and regularly monitored for quality control.

Statistical analyses. Baseline characteristics of individuals according to T2D status were compared using Student's $t$-test and Pearson's Chi-square test for continuous and categorical variables, respectively. We determined the association between changes in MetS components over a two-year period and the incidence of T2D during four years of follow-up using Cox proportional hazards regression analysis. Hazard ratios (HRs) and 95\% confidence intervals (CIs) were calculated. HRs were adjusted for sociodemographic characteristics. Model 1 was adjusted for age and sex. Model 2 was adjusted for age, sex, alcohol consumption, smoking status, physical activity, and BMI. All statistical tests were two-sided, and $p<0.05$ was considered significant. All analyses were performed using the Statistical Analysis System statistical software package, version 9.4 (SAS Institute Inc., Cary, NC, USA).

Ethics approval and consent to participate. This study was approved by the NHIS inquiry commission and adhered to the tenets of the Declaration of Helsinki for biomedical research. Informed consent was not required because the national insurance claim data were deidentification for the analysis. This study was also approved by the Institutional Review Board of The Catholic University of Korea (No. SC18ZESIO047).

\section{Results}

General baseline characteristics. During a median follow-up of 4.087 years, a total of 848,859 individuals were diagnosed with T2D. Table 1 presents baseline demographics of the study population according to new-onset T2D status. Individuals who developed T2D were more likely to be older and males with higher BMI, WC, TC, BP, and FPG values than those without T2D. They also had higher rates of MetS (41.15\% vs. 18.65\%), abdominal obesity ( $28.31 \%$ vs. $15.63 \%$ ), elevated triglycerides ( $45.51 \%$ vs. $29.48 \%)$, reduced HDL-cholesterol (37.36\% vs. $23.15 \%)$, elevated BP (58\% vs. $37.08 \%$ ), and elevated FPG (37.94\% vs. $22.28 \%$ ).

Changes in the number of MetS components and the risk of T2D. Table 2 shows associations between changes in number of MetS components and risk of T2D. For individuals with the same number of MetS components at baseline examination, HR for incident T2D was lower for lower numbers of MetS components at the second visit than at baseline, and vice versa, it was higher as the number increased. After adjusting for age, sex, alcohol drinking, smoking status, exercise, and BMI, HR for incident T2D was 0.51 (95\% CI: 0.497-0.522) for individuals with four to five MetS components at baseline and zero to one component at the second time point. It was 0.268 (95\% CI: 0.266-0.271) for those with zero to one component at baseline and zero to one component at the second visit compared to those with four to five components at both time points (Table 2).

HRs for incident T2D decreased as the number of MetS components at baseline decreased. Likewise, the lower the number at the second visit, the lower the risk of incident T2D (both $p$ for trend $<0.0001$ ). The risk of T2D increased with the number of MetS components at both time points ( $p$ for trend $<0.0001$, Fig. 2).

Multivariable hazard ratios were adjusted for age, sex, alcohol drinking, smoking status, physical activity, and BMI. 


\begin{tabular}{|c|c|c|c|c|c|c|c|}
\hline \multicolumn{2}{|c|}{ No. of components } & \multirow[b]{2}{*}{ No. } & \multirow[b]{2}{*}{ Cases, $n$} & \multirow{2}{*}{\begin{tabular}{|l|} 
Follow-up \\
duration \\
(person-years)
\end{tabular}} & \multirow{2}{*}{$\begin{array}{l}\text { Incidence rate } \\
\text { (per } 1,000 \\
\text { person-years) }\end{array}$} & \multicolumn{2}{|l|}{ HR (95\% CI) } \\
\hline Baseline & \begin{tabular}{|l|}
$2^{\text {nd }}$ \\
visit
\end{tabular} & & & & & MODEL1 & MODEL2 \\
\hline \multirow{4}{*}{4,5} & 4,5 & 607370 & 323806 & 2601446.3 & 124.472 & 1 (ref.) & 1 (ref.) \\
\hline & 3 & 272829 & 87487 & 1225757.06 & 71.374 & $0.727(0.718,0.735)$ & $0.744(0.735,0.752)$ \\
\hline & 2 & 147504 & 35700 & 677738.11 & 52.675 & $0.598(0.589,0.608)$ & $0.617(0.607,0.626)$ \\
\hline & 0,1 & 67926 & 10613 & 319037.6 & 33.266 & $0.482(0.47,0.494)$ & $0.51(0.497,0.522)$ \\
\hline \multirow{4}{*}{3} & 4,5 & 336591 & 114105 & 1493535.62 & 76.399 & $0.851(0.842,0.86)$ & $0.864(0.855,0.873)$ \\
\hline & 3 & 488771 & 116144 & 2239703.01 & 51.857 & $0.637(0.63,0.643)$ & $0.66(0.653,0.667)$ \\
\hline & 2 & 404229 & 67614 & 1897444.11 & 35.634 & $0.511(0.505,0.517)$ & $0.534(0.528,0.54)$ \\
\hline & 0,1 & 283606 & 30233 & 1358389.06 & 22.257 & $0.396(0.39,0.402)$ & $0.424(0.418,0.431)$ \\
\hline \multirow{4}{*}{2} & 4,5 & 210854 & 55861 & 950753.22 & 58.754 & $0.761(0.751,0.771)$ & $0.777(0.768,0.787)$ \\
\hline & 3 & 464831 & 79523 & 2168358.98 & 36.674 & $0.56(0.554,0.566)$ & $0.583(0.577,0.59)$ \\
\hline & 2 & 779499 & 103786 & 3700729.29 & 28.045 & $0.44(0.436,0.445)$ & $0.467(0.462,0.471)$ \\
\hline & 0,1 & 910113 & 69895 & 4415757.5 & 15.829 & $0.33(0.326,0.333)$ & $0.359(0.355,0.363)$ \\
\hline \multirow{4}{*}{0,1} & 4,5 & 107365 & 20352 & 491251.63 & 41.429 & $0.673(0.661,0.684)$ & $0.701(0.689,0.713)$ \\
\hline & 3 & 376749 & 44911 & 1781363.82 & 25.212 & $0.478(0.472,0.484)$ & $0.507(0.501,0.513)$ \\
\hline & 2 & 1033834 & 82456 & 4986623.56 & 16.535 & $0.36(0.356,0.363)$ & $0.388(0.384,0.392)$ \\
\hline & 0,1 & 4835414 & 196776 & 23793248.67 & 8.27 & $0.239(0.237,0.241)$ & $0.268(0.266,0.271)$ \\
\hline
\end{tabular}

Table 2. Hazard Ratios (95\% CI) for Incident Type 2 Diabetes According to Changes in Number of Metabolic Syndrome Components from Baseline to the Second Visit. Model 1 was adjusted for age and sex. Model 2 was adjusted for age, sex, alcohol consumption, smoking status, physical activity, and BMI.

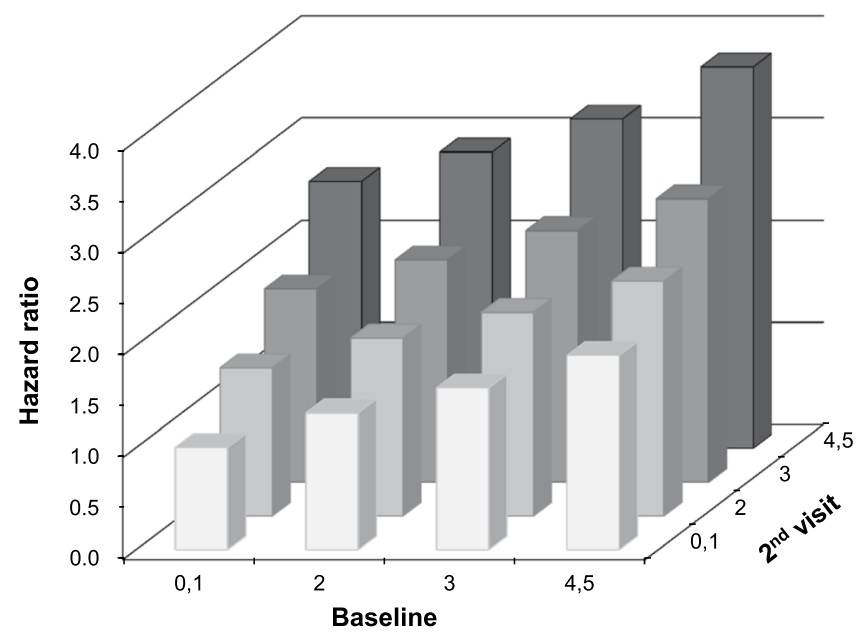

Figure 2. Hazard ratios (95\% CI) for incident diabetes according to changes in number of metabolic syndrome components. The risk of diabetes was increased with the number of metabolic syndrome components at baseline examination and the second visit $(p$ for trend $<0.0001)$.

Changes in MetS and its components and the risk of T2D. Table 3 shows associations of changes in MetS and its components with the risk of T2D. The incidence rate of incident T2D was the highest in individuals with MetS or its components at both time points. It was the lowest in those without MetS or its components at both time points. Individuals with MetS or its components at baseline but without them at the second visit showed a decreased incidence of T2D (Table 3). HR for incident T2D was the lowest in individuals without MetS or its components at both time points. It was higher in those without MetS or its components at baseline and with them at the second visit than those with them at baseline but without them at the second visit.

Figure 3 describes risk reduction of diabetes according to improvement in MetS and its individual components. Multivariable-adjusted HR for incident T2D was 0.645 (95\% CI: 0.64-0.65) in individuals with reduced number of MetS components compared to those with MetS at both time points. HRs for incident T2D were 0.54 (95\% CI: 0.537-0.544) for those with improvement in elevated fasting glucose, 0.735 (95\% CI: 0.73-0.741) for those with improvement in elevated triglycerides, 0.763 (95\% CI: $0.757-0.769)$ for those with improvement in reduced HDL-cholesterol, 0.746 (95\% CI: 0.741-0.752) for those with improvement in elevated blood pressure, and 0.92 (95\% CI: 0.912-0.928) for those with improvement in elevated waist circumference compared to those with each component of MetS at both time points (Fig. 3). 


\begin{tabular}{|c|c|c|c|c|c|c|c|c|}
\hline \multirow[b]{2}{*}{ MetS and its components } & \multicolumn{2}{|l|}{ Status } & \multirow[b]{2}{*}{ No. } & \multirow[b]{2}{*}{ Cases, $\mathbf{n}$} & \multirow[b]{2}{*}{$\begin{array}{l}\text { Follow-up } \\
\text { duration }\end{array}$} & \multirow[b]{2}{*}{ Incidence rate } & \multicolumn{2}{|l|}{ HR $(95 \% \mathrm{CI})$} \\
\hline & Baseline & $\begin{array}{l}2^{\text {nd }} \\
\text { visit }\end{array}$ & & & & & MODEL1 & MODEL2 \\
\hline \multirow{4}{*}{ Metabolic syndrome } & Yes & Yes & 1348002 & 258335 & 4858948 & 53.1669 & 1(ref.) & 1(ref.) \\
\hline & \begin{tabular}{|l|} 
Yes \\
\end{tabular} & No & 858647 & 90967 & 3278848 & 27.7436 & $0.609(0.605,0.614)$ & $0.645(0.64,0.65)$ \\
\hline & No & Yes & 1105518 & 134303 & 4152818 & 32.3402 & $0.727(0.722,0.732)$ & $0.76(0.755,0.765)$ \\
\hline & No & No & 7494549 & 365254 & 29490297 & 12.3856 & $0.374(0.372,0.376)$ & $0.43(0.427,0.432)$ \\
\hline \multirow{4}{*}{ Elevated fasting glucose } & Yes & Yes & 6808054 & 373209 & 26699935 & 47.674 & 1(ref.) & 1(ref.) \\
\hline & Yes & No & 1365996 & 118208 & 5281524 & 22.3814 & $0.52(0.516,0.523)$ & $0.54(0.537,0.544)$ \\
\hline & No & Yes & 1457786 & 153601 & 5523729 & 27.8075 & $0.642(0.638,0.646)$ & $0.656(0.651,0.66)$ \\
\hline & No & No & 6808054 & 373209 & 26699935 & 13.9779 & $0.375(0.373,0.377)$ & $0.404(0.401,0.406)$ \\
\hline \multirow{4}{*}{ Elevated triglycerides } & Yes & Yes & 6144925 & 334902 & 24034506 & 34.7754 & 1(ref.) & 1(ref.) \\
\hline & Yes & No & 1119128 & 98029 & 4315085 & 22.7177 & $0.689(0.684,0.694)$ & $0.735(0.73,0.741)$ \\
\hline & No & Yes & 1340144 & 127679 & 5142447 & 24.8285 & $0.777(0.771,0.782)$ & $0.815(0.81,0.821)$ \\
\hline & No & No & 6144925 & 334902 & 24034506 & 13.9342 & $0.51(0.507,0.512)$ & $0.588(0.585,0.591)$ \\
\hline \multirow{4}{*}{ Reduced HDL-cholesterol } & Yes & Yes & 6950041 & 394884 & 27259745 & 38.6107 & 1(ref.) & 1(ref.) \\
\hline & Yes & No & 1072221 & 95340 & 4122993 & 23.124 & $0.725(0.72,0.731)$ & $0.763(0.757,0.769)$ \\
\hline & No & Yes & 1233966 & 136825 & 4653389 & 29.4033 & $0.881(0.875,0.887)$ & $0.91(0.904,0.917)$ \\
\hline & No & No & 6950041 & 394884 & 27259745 & 14.486 & $0.555(0.552,0.558)$ & $0.621(0.617,0.624)$ \\
\hline \multirow{4}{*}{ Elevated blood pressure } & Yes & Yes & 5211154 & 244220 & 20463164 & 36.4587 & 1(ref.) & 1(ref.) \\
\hline & Yes & No & 1206373 & 86152 & 4717310 & 18.263 & $0.695(0.69,0.7)$ & $0.746(0.741,0.752)$ \\
\hline & No & Yes & 1410607 & 112337 & 5460447 & 20.5729 & $0.76(0.755,0.765)$ & $0.799(0.794,0.804)$ \\
\hline & No & No & 5211154 & 244220 & 20463164 & 11.9346 & $0.553(0.55,0.556)$ & $0.638(0.635,0.642)$ \\
\hline \multirow{4}{*}{ Elevated waist circumference } & Yes & Yes & 1157171 & 167263 & 4307384 & 38.8317 & 1(ref.) & 1(ref.) \\
\hline & Yes & No & 768305 & 81784 & 2911413 & 30.2415 & $0.766(0.759,0.772)$ & $0.92(0.912,0.928)$ \\
\hline & No & Yes & 639494 & 73017 & 2414465 & 28.0908 & $0.786(0.78,0.793)$ & $0.904(0.896,0.912)$ \\
\hline & No & No & \begin{tabular}{|l|}
8241746 \\
\end{tabular} & 526795 & 32147649 & 16.3867 & $0.553(0.55,0.556)$ & $0.794(0.788,0.8)$ \\
\hline
\end{tabular}

Table 3. Multivariable-adjusted Hazard Ratios for Type 2 Diabetes According to Changes in Metabolic Syndrome and Its Components. Model 1 was adjusted for age and sex. Model 2 was adjusted for age, sex, alcohol consumption, smoking status, physical activity, and BMI.

\section{Discussion}

In this prospective study of 10,806,716 adults, we found that changes in MetS and its components were associated with the risk of incident T2D. The risk of T2D was significantly lowered with a decrease in the number of MetS components at both time points regardless of the type of MetS component. In addition, improving MetS and its components reduced the risk of $\mathrm{T} 2 \mathrm{D}$.

The incidence of MetS has increased markedly in Korea ${ }^{17}$. Numerous epidemiological studies have demonstrated that MetS and its components can predict the development of T2D ${ }^{18}$. In the current longitudinal study, we found that improvement in MetS and its components was associated with a reduced risk of T2D. After adjusting for confounding factors such as age, sex, alcohol consumption, smoking status, exercise, and BMI, we found a $36 \%$ decrease in diabetes risk among individuals with reduced number of MetS components at the second visit, a $46 \%$ decrease with improvement in elevated FPG, a 27\% decrease with improvement in elevated triglycerides, a $25 \%$ decrease with improvement in elevated blood pressure, a $24 \%$ decrease with improvement in reduced HDL-cholesterol, and a $8 \%$ decrease with improvement in abdominal obesity. Although it was not feasible to compare relative risks of changes in MetS and its components directly, the risk of diabetes was further reduced in individuals with improvement of elevated FPG than in those with improvement in MetS. Among MetS components, improvement in elevated FPG greatly reduced the risk of T2D.

Elevated FPG level has been comprehensively investigated as a core component in models for risk assessment of T2 ${ }^{19,20}$. Analysis of the Tehran Lipid and Glucose Study showed a positive association between changes in FPG levels and the incidence of T2D 21 . As expected, our results indicated that changes in FPG levels were most strongly associated with the development of T2D. It is well known that elevated FPG is a predictor of progression to diabetes ${ }^{22}$. Some studies have reported that individuals with MetS manifest a higher risk of developing diabetes than those with elevated FPG $^{23}$ whereas other studies have indicated that elevated FPG is more effective than MetS for predicting incident diabetes ${ }^{24}$. In addition, it has been suggested that MetS is less strongly associated with the risk of T2D in Asian populations than in Western populations ${ }^{25}$. This discrepancy may result from differences in the degree of insulin resistance of these populations ${ }^{26}$. The current study showed that elevated FPG was a more potent component than MetS for the prevention of T2D.

In this study, we found that all MetS components were potentially related to the risk of incident T2D. Elevated triglyceride and reduced HDL-cholesterol are common dyslipidemic features accompanying T2D ${ }^{27}$. Some studies have shown that changes in fasting triglyceride levels are predictive of T2D, independent of traditional risk factors ${ }^{28}$. Reduced HDL-cholesterol is associated with an increased risk of T2D ${ }^{29}$. An increase in triglyceride level, particularly when it is accompanied by low HDL-cholesterol, has consistently been shown to be a surrogate 


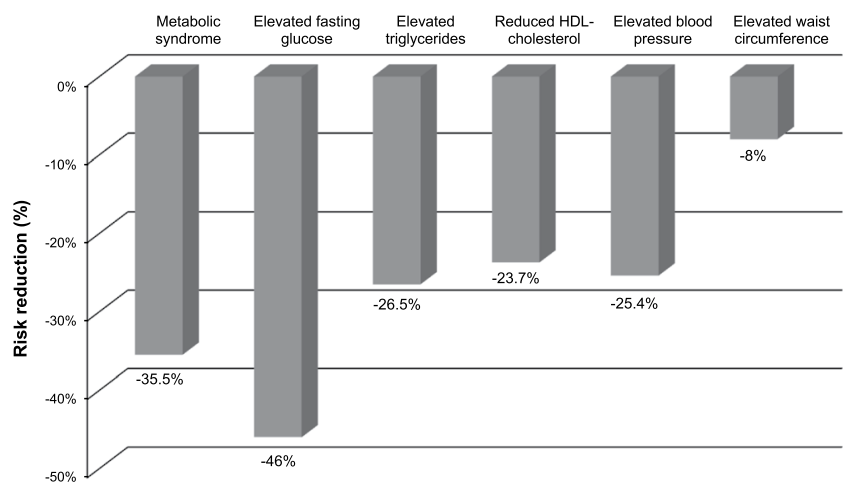

Figure 3. Risk reduction based on improvement in metabolic syndrome and its components.

marker of insulin resistance ${ }^{30}$ which is a strong predisposing condition for T2D. Elevated BP has been identified as a risk factor for $\mathrm{T} 2 \mathrm{D}^{31}$. A strong association between abdominal obesity and the development of T2D has been recognized ${ }^{32}$. Interestingly, after adjusting for traditional risk factors such as BMI, we found that change in abdominal obesity remained a significant predictor of T2D. Indeed, when we analyzed the contribution of each MetS component, our findings showed an independent correlation of each component with diabetes risk. Thus, improving each component of MetS can reduce the risk of diabetes.

Lifestyle factors such as diet and physical activity can strongly influence metabolic parameters such as FPG, triglycerides, HDL-cholesterol, BP, and waist circumference ${ }^{33}$. Clinical trials have shown that lifestyle modifications can substantially reduce the risk of developing diabetes ${ }^{34}$. Smoking and excessive alcohol consumption are important risk factors for the development of MetS and its components ${ }^{35,36}$. The level of metabolic parameters at a single time point may inaccurately reflect the long-term risk of T2D. Therefore, we investigated the association of T2D with changes in metabolic parameters at two different time points in the current study. The effect of controlling MetS and its components on the development of T2D was determined after adjusting for age, sex, alcohol consumption, smoking status, physical activity, and BMI. The present longitudinal study indicated that changes in MetS and its components remained independent determinants of diabetes risk even after adjusting for lifestyle factors.

Strengths of the present study include its large sample size and a prospective cohort study design which ensured a thorough follow-up. In addition, the level of metabolic parameters at a single time point may inaccurately reflect the long-term risk of developing diabetes. We demonstrate a significant association of changes in MetS and its components with the risk of T2D. Our findings could be a cornerstone for other studies to explore the impact of changes in MetS on the risk for T2D-related outcomes. However, this study has several limitations. First, the four-year follow-up period was relatively short. Results might have been more significant with a longer follow-up. Second, diagnostic criteria for MetS vary between populations belonging to different ethnicities. Results may differ according to other diagnostic criteria. Nevertheless, the modified criteria of NCEP ATP III have been more strongly associated with T2D than Internal Diabetes Federation-defined MetS in Korean adults ${ }^{37}$. Third, the incidence of T2D might have been underestimated because individuals with undiagnosed T2D or those who failed to visit a hospital during the study period were excluded from this study. Fourth, this analysis relied on the NHIS DB. Basic laboratory tests, including fasting glucose, are performed in this health check-up program. Therefore, we could not obtain other laboratory values, such as glycosylated hemoglobin levels. In addition, these data do not include uninsured events because the data are basically made for claim purposes and only represent the services covered by the NHIS. For these reasons, discrepancies between the actual diagnosis and the NHIS DB might be possible. However, the operational definitions of T2D, which have been used in several studies, are appropriately validated to minimize inconsistent and inaccurate results. The NHIS DB represents the entire Korean population; therefore, it can be used in a population-based nationwide study for T2DM in Korea. Finally, although we adjusted for potential confounding factors, lifestyle factors that were unaccounted for in this study might have mediated changes in MetS. Further high-quality studies are needed to corroborate these results.

\section{Conclusions}

In this large-scale cohort study, we found that longitudinal changes in MetS and its components were significantly associated with the development of T2D. Improvement in MetS and its components reduced the risk of diabetes, independent of lifestyle factors. Changes in each of MetS components could predict the development of T2D. In Korea, improvement in elevated FPG was more strongly related to a reduced risk of T2D than improvement in MetS. Although our present study could not establish causal relationships, the modifying effect of changes in MetS and its components on diabetes risk may provide new insights into strategies to prevent T2D.

\section{Data availability}

The data that support the findings of this study are available from the National Health Insurance Service but restrictions apply to the availability of these data, which were used under license for the current study, and so are not publicly available. Access to the dataset can be obtained through the Health Insurance Data Service home page (http://nhiss.nhis.or.kr). 
Received: 16 August 2019; Accepted: 21 January 2020; Published online: 11 February 2020

\section{References}

1. Wu, Y., Ding, Y., Tanaka, Y. \& Zhang, W. Risk factors contributing to type 2 diabetes and recent advances in the treatment and prevention. Int J Med Sci 11, 1185-1200, https://doi.org/10.7150/ijms.10001 (2014).

2. Siegel, K. R. et al. Prevalence of Major Behavioral Risk Factors for Type 2 Diabetes. Diabetes Care 41, 1032-1039, https://doi. org/10.2337/dc17-1775 (2018).

3. World Health Organization. Global Report on Diabetes (World Health Organization, 2016).

4. Simmons, R. K. et al. The metabolic syndrome: useful concept or clinical tool? Report of a WHO Expert Consultation. Diabetologia 53, 600-605, https://doi.org/10.1007/s00125-009-1620-4 (2010).

5. Huang, P. L. A comprehensive definition for metabolic syndrome. Dis Model Mech 2, 231-237, https://doi.org/10.1242/dmm.001180 (2009).

6. Sattar, N. et al. Can metabolic syndrome usefully predict cardiovascular disease and diabetes? Outcome data from two prospective studies. Lancet 371, 1927-1935, https://doi.org/10.1016/s0140-6736(08)60602-9 (2008).

7. Hanson, R. L., Imperatore, G., Bennett, P. H. \& Knowler, W. C. Components of the "metabolic syndrome" and incidence of type 2 diabetes. Diabetes 51, 3120-3127, https://doi.org/10.2337/diabetes.51.10.3120 (2002).

8. Nakanishi, N. et al. Components of the metabolic syndrome as predictors of cardiovascular disease and type 2 diabetes in middleaged Japanese men. Diabetes Res Clin Pract 64, 59-70, https://doi.org/10.1016/j.diabres.2003.10.001 (2004).

9. Shin, J. A. et al. Metabolic syndrome as a predictor of type 2 diabetes, and its clinical interpretations and usefulness. J Diabetes Investig 4, 334-343, https://doi.org/10.1111/jdi.12075 (2013).

10. Grundy, S. M. et al. Diagnosis and management of the metabolic syndrome: an American Heart Association/National Heart, Lung, and Blood Institute Scientific Statement. Circulation 112, 2735-2752, https://doi.org/10.1161/CIRCULATIONAHA.105.169404 (2005).

11. Cheol Seong, S. et al. Data Resource Profile: The National Health Information Database of the National Health Insurance Service in South Korea. Int J Epidemiol 46, 799-800, https://doi.org/10.1093/ije/dyw253 (2017).

12. Song, S. O. et al. Background and data configuration process of a nationwide population-based study using the korean national health insurance system. Diabetes Metab J 38, 395-403, https://doi.org/10.4093/dmj.2014.38.5.395 (2014).

13. Ko, S.-H. et al. Past and Current Status of Adult Type 2 Diabetes Mellitus Management in Korea: A National Health Insurance Service Database Analysis. Diabetes Metab J 42, 93-100 (2018).

14. Alberti, K. G. et al. Harmonizing the metabolic syndrome: a joint interim statement of the International Diabetes Federation Task Force on Epidemiology and Prevention; National Heart, Lung, and Blood Institute; American Heart Association; World Heart Federation; International Atherosclerosis Society; and International Association for the Study of Obesity. Circulation 120, 1640-1645, https://doi.org/10.1161/CIRCULATIONAHA.109.192644 (2009).

15. Lee, S. Y. et al. Appropriate waist circumference cutoff points for central obesity in Korean adults. Diabetes Res Clin Pract 75, 72-80, https://doi.org/10.1016/j.diabres.2006.04.013 (2007).

16. Notification No. 2018-264, The Ministry of health and Welfare, Heath checkups implementation standard, http://www.mohw.go.kr/ upload/viewer/skin/doc.html?fn=1545354411060_20181221100651.hwp\&rs=/upload/viewer/result/201912/ (2019).

17. Lee, S. E. et al. Trends in the prevalence of metabolic syndrome and its components in South Korea: Findings from the Korean National Health Insurance Service Database (2009-2013). PLoS One 13, e0194490, https://doi.org/10.1371/journal.pone.0194490 (2018).

18. Wang, K. et al. Metabolic Syndrome and Its Components Predict the Risk of Type 2 Diabetes Mellitus in the Mainland Chinese: A 3-Year Cohort Study. Int J Endocrinol 2018, 9376179, https://doi.org/10.1155/2018/9376179 (2018).

19. Noble, D., Mathur, R., Dent, T., Meads, C. \& Greenhalgh, T. Risk models and scores for type 2 diabetes: systematic review. BMJ 343, d7163, https://doi.org/10.1136/bmj.d7163 (2011).

20. Tabák, A. G., Herder, C., Rathmann, W., Brunner, E. J. \& Kivimäki, M. Prediabetes: a high-risk state for diabetes development. Lancet 379, 2279-2290, https://doi.org/10.1016/S0140-6736(12)60283-9 (2012).

21. Mozaffary, A. et al. Change in fasting plasma glucose and incident type 2 diabetes mellitus: results from a prospective cohort study. BMJ Open 6, e010889, https://doi.org/10.1136/bmjopen-2015-010889 (2016).

22. Nichols, G. A., Hillier, T. A. \& Brown, J. B. Progression from newly acquired impaired fasting glusose to type 2 diabetes. Diabetes Care 30, 228-233, https://doi.org/10.2337/dc06-1392 (2007).

23. Lorenzo, C., Williams, K., Hunt, K. J. \& Haffner, S. M. The National Cholesterol Education Program - Adult Treatment Panel III, International Diabetes Federation, and World Health Organization definitions of the metabolic syndrome as predictors of incident cardiovascular disease and diabetes. Diabetes Care 30, 8-13, https://doi.org/10.2337/dc06-1414 (2007).

24. Cameron, A. J. et al. The metabolic syndrome as a tool for predicting future diabetes: the AusDiab study. J Intern Med 264, 177-186, https://doi.org/10.1111/j.1365-2796.2008.01935.x (2008).

25. Mukai, N. et al. Impact of metabolic syndrome compared with impaired fasting glucose on the development of type 2 diabetes in a general Japanese population: the Hisayama study. Diabetes Care 32, 2288-2293, https://doi.org/10.2337/dc09-0896 (2009).

26. Jensen, C. C. et al. Beta-cell function is a major contributor to oral glucose tolerance in high-risk relatives of four ethnic groups in the U.S. Diabetes 51, 2170-2178, https://doi.org/10.2337/diabetes.51.7.2170 (2002).

27. Goldberg, I. J. Clinical review 124: Diabetic dyslipidemia: causes and consequences. J Clin Endocrinol Metab 86, 965-971, https:// doi.org/10.1210/jcem.86.3.7304 (2001).

28. Tirosh, A. et al. Changes in triglyceride levels over time and risk of type 2 diabetes in young men. Diabetes Care 31, 2032-2037, https://doi.org/10.2337/dc08-0825 (2008).

29. Carmena, R. Type 2 diabetes, dyslipidemia, and vascular risk: rationale and evidence for correcting the lipid imbalance. Am Heart $J$ 150, 859-870, https://doi.org/10.1016/j.ahj.2005.04.027 (2005).

30. McLaughlin, T. et al. Is there a simple way to identify insulin-resistant individuals at increased risk of cardiovascular disease? Am J Cardiol 96, 399-404, https://doi.org/10.1016/j.amjcard.2005.03.085 (2005).

31. Kim, M. J., Lim, N. K., Choi, S. J. \& Park, H. Y. Hypertension is an independent risk factor for type 2 diabetes: the Korean genome and epidemiology study. Hypertens Res 38, 783-789, https://doi.org/10.1038/hr.2015.72 (2015).

32. Freemantle, N., Holmes, J., Hockey, A. \& Kumar, S. How strong is the association between abdominal obesity and the incidence of type 2 diabetes? Int J Clin Pract 62, 1391-1396, https://doi.org/10.1111/j.1742-1241.2008.01805.x (2008).

33. Huang, J. H. et al. Lifestyle Factors and Metabolic Syndrome among Workers: The Role of Interactions between Smoking and Alcohol to Nutrition and Exercise. Int J Environ Res Public Health 12, 15967-15978, https://doi.org/10.3390/ijerph121215035 (2015).

34. Knowler, W. C. et al. Reduction in the incidence of type 2 diabetes with lifestyle intervention or metformin. N Engl J Med 346, 393-403, https://doi.org/10.1056/NEJMoa012512 (2002).

35. Oh, S. W. et al. Association between cigarette smoking and metabolic syndrome: the Korea National Health and Nutrition Examination Survey. Diabetes Care 28, 2064-2066, https://doi.org/10.2337/diacare.28.8.2064 (2005). 
36. Lee, K. W., Park, B. J., Kang, H. T. \& Lee, Y. J. Alcohol-drinking patterns and metabolic syndrome risk: the 2007 Korean National Health and Nutrition Examination Survey. Alcohol 45, 499-505, https://doi.org/10.1016/j.alcohol.2011.01.001 (2011).

37. Kim, H. M., Kim, D. J., Jung, I. H., Park, C. \& Park, J. Prevalence of the metabolic syndrome among Korean adults using the new International Diabetes Federation definition and the new abdominal obesity criteria for the Korean people. Diabetes Res Clin Pract 77, 99-106, https://doi.org/10.1016/j.diabres.2006.10.009 (2007).

\section{Acknowledgements}

This research was supported by the Basic Science Research Program through the National Research Foundation of Korea (NRF) funded by the Ministry of Education (2018R1D1A1B07049079).

\section{Author contributions}

M.K.L., K.D.H. and H.S.K. contributed to the study design, analysis, and interpretation of data. M.K.L. drafted and edited the manuscript. H.S.K. provided supervision and revised the manuscript. K.D.H. performed the statistical analysis of data. M.K.K., E.S.K., E.S.K. and G.E.N. contributed to the interpretation of the results. All authors have read and approved the final manuscript.

\section{Competing interests}

The authors declare no competing interests.

\section{Additional information}

Correspondence and requests for materials should be addressed to H.-S.K.

Reprints and permissions information is available at www.nature.com/reprints.

Publisher's note Springer Nature remains neutral with regard to jurisdictional claims in published maps and institutional affiliations.

Open Access This article is licensed under a Creative Commons Attribution 4.0 International License, which permits use, sharing, adaptation, distribution and reproduction in any medium or format, as long as you give appropriate credit to the original author(s) and the source, provide a link to the Creative Commons license, and indicate if changes were made. The images or other third party material in this article are included in the article's Creative Commons license, unless indicated otherwise in a credit line to the material. If material is not included in the article's Creative Commons license and your intended use is not permitted by statutory regulation or exceeds the permitted use, you will need to obtain permission directly from the copyright holder. To view a copy of this license, visit http://creativecommons.org/licenses/by/4.0/.

(c) The Author(s) 2020 